COMMUNICATIONS IN

ANALYSIS AND GEOMETRY

Volume 14, Number 2, 387-410, 2006

\title{
On the Euler numbers of certain moduli spaces of curves and points
}

\author{
WeI-PING Li ${ }^{1}$ AND Zhenbo QIN ${ }^{2}$
}

\begin{abstract}
We determine the topological Euler number of certain moduli space of 1-dimensional closed subschemes in a smooth projective variety which admits a Zariski-locally trivial fibration with 1-dimensional fibers. The main approach is to use virtual Hodge polynomials and torus actions. The results might shed some light on the corresponding Donaldson-Thomas invariants.
\end{abstract}

\section{Introduction.}

Recently, there have been surging interests in studying the moduli spaces of 1-dimensional closed subschemes in a smooth projective variety. The motivation comes from Donaldson-Thomas theory and its interplay with Gromov-Witten theory and Gopakumar-Vafa invariants [13], [14], [11], [17] (see the references there for other papers). Donaldson-Thomas theory was introduced in [5], [18] via integrals over the moduli spaces of semistable sheaves and via the theory of virtual fundamental cycles. It was further developed by Maulik-Pandharipande [15] and Jun Li. The moduli spaces of 1-dimensional closed subschemes in a smooth projective variety can be naturally regarded as the moduli spaces of rank-1 stable sheaves over the variety.

In [13], [14], several interesting conjectures regarding the interplay among Donaldson-Thomas theory, Gromov-Witten theory and Gopakumar-Vafa invariants have been proposed for 3 -folds. In particular, it was conjectured (see the Conjecture 2 in both papers) that the reduced partition function (with a formal variable $q$ ) for the Donaldson-Thomas invariants is a rational function of $q$, and is invariant under the transformation $q \rightarrow 1 / q$ when the 3 -fold is Calabi-Yau. It is possible that, at least in the case of Calabi-Yau 3folds, the Donaldson-Thomas invariants are closely related to the topological

\footnotetext{
${ }^{1}$ Partially supported by the grant HKUST6114/02P.

${ }^{2}$ Partially supported by an NSF grant.
} 
Euler numbers of the corresponding moduli spaces of 1-dimensional closed subschemes. Hence, we propose the following analogue to the Conjecture 2 in [13], [14].

Conjecture 1.1. Let $X$ be a smooth projective complex variety. Let $\mathfrak{I}_{n}(X, \beta)$ be the moduli space of 1-dimensional closed subschemes $Z$ of $X$ satisfying (3.1), and let $X^{[n]}=\mathfrak{I}_{n}(X, 0)$ be the Hilbert scheme of length-n 0 -dimensional closed subschemes of $X$. Then the reduced partition function for the Euler numbers

$$
\frac{\sum_{n} \chi\left(\Im_{n}(X, \beta)\right) q^{n}}{\sum_{n} \chi\left(X^{[n]}\right) q^{n}}
$$

is a rational function of $q$, and is invariant under $q \rightarrow 1 / q$ when $K_{X}=0$.

While Donaldson-Thomas invariants are difficult to calculate in general, there exist many effective methods to compute Euler numbers. In this paper, we verify Conjecture 1.1 under certain assumptions. Specifically, we assume that $X$ admits a Zariski-locally trivial fibration $\mu: X \rightarrow S$ where $S$ is smooth, the fibers are smooth irreducible curves of genus- $g$, and $\beta \in H_{2}(X ; \mathbb{Z})$ is the class of a fiber. An element in the moduli space $\mathfrak{I}_{(1-g)+n}(X, \beta)$ consists of a fiber of $\mu$ together with some points (possibly embedded in the same fiber). For simplicity, let

$$
\mathfrak{M}_{n}=\mathfrak{I}_{(1-g)+n}(X, \beta) \text {. }
$$

Theorem 1.2. Let $r=\operatorname{dim}(X) \geq 2$, and $P_{r}(n)$ (respectively, $\left.\widetilde{P}_{r}(n)\right)$ be the number of $r$-dimensional (respectively, punctual $r$-dimensional) partitions of n. Then,

$$
\sum_{n=0}^{+\infty} \chi\left(\mathfrak{M}_{n}\right) q^{n}=\sum_{n=0}^{+\infty} \chi\left(X^{[n]}\right) q^{n} \cdot \chi(S) \cdot\left(\frac{\sum_{n=0}^{+\infty} \widetilde{P}_{r}(n) q^{n}}{\sum_{n=0}^{+\infty} P_{r}(n) q^{n}}\right)^{2-2 g} .
$$

We remark that the Euler number of the Hilbert scheme $X^{[n]}$ has been determined by Göttsche and Cheah $[7,2]$, and $\chi\left(\mathfrak{M}_{1}\right)$ can be computed from the Lemma 1 in [11] where the structure of $\mathfrak{M}_{1}$ has been described. Also, we refer to Definition 7.1 for the notion of $r$-dimensional (respectively, punctual $r$-dimensional) partitions of $n$. Furthermore, we conjecture (see Conjecture 7.4) that

$$
\frac{\sum_{n=0}^{+\infty} \widetilde{P}_{r}(n) q^{n}}{\sum_{n=0}^{+\infty} P_{r}(n) q^{n}}=\frac{1}{(1-q)^{r-2}}
$$


This formula is trivially true when $r=2$. The case $r=3$ is proved in Lemma 7.5. Note that $K_{X}=0$ forces $g=1$ under our assumption about $\mu$.

Corollary 1.3. Conjecture 1.1 is true when $2 \leq r \leq 3$ or $K_{X}=0$.

Our main idea to prove Theorem 1.2 is to use virtual Hodge polynomials, Cheah's combinatorial arguments [2], and torus actions. More precisely, we decompose the moduli space $\mathfrak{M}_{n}$ into a disjoint union of locally closed subsets, and prove that there exist bijective morphisms between these locally closed subsets and certain spaces constructed from the local model $\mathbb{C}^{r-1} \times C$ of the fibration $\mu: X \rightarrow S$, where $C$ denotes a fixed fiber of $\mu$. The spaces constructed from the local model $\mathbb{C}^{r-1} \times C$ consist of Zariski-locally trivial fibrations involving simpler objects. Our proofs here are parallel to those in [7]. Similar results hold for the Hilbert scheme $X^{[n]}$. Since virtual Hodge polynomials are preserved under decompositions and bijective morphisms, these preparations enable us to reduce the computation of the virtual Hodge polynomial $e\left(\mathfrak{M}_{n} ; s, t\right)$ to those of

$$
\mathfrak{M}_{n, C_{0}}^{\mathbb{C}^{r-1} \times C}, \quad\left(\mathbb{C}^{r-1} \times C\right)_{C_{0}}^{[n]}
$$

(see Definition 3.3 and Remark 3.4 for the notations), where $C_{0}=\{O\} \times C$ and $O$ is the origin of $\mathbb{C}^{r-1}$. Applying Cheah's combinatorial arguments, we show that the virtual Hodge polynomials of $\mathfrak{M}_{n, C_{0}}^{\mathbb{C}^{r-1} \times C}$ and $\left(\mathbb{C}^{r-1} \times C\right)_{C_{0}}^{[n]}$ can be further reduced to those of the corresponding punctual spaces:

$$
\mathfrak{M}_{n, L, O}^{\mathbb{C}^{r}}, \quad \operatorname{Hilb}^{n}\left(\mathbb{C}^{r}, O\right)
$$

where $L$ denotes a coordinate line in $\mathbb{C}^{r}$. Using the well-known fact that $e(\cdot ; 1,1)=\chi(\cdot)$, we obtain a formula for $\chi\left(\mathfrak{M}_{n}\right)$ in terms of the Euler numbers of $\mathfrak{M}_{n, L, O}^{\mathbb{C}^{r}}$ and $\operatorname{Hilb}^{n}\left(\mathbb{C}^{r}, O\right.$ ) (see Proposition 6.2). Finally, we take suitable torus actions on $\mathfrak{M}_{n, L, O}^{\mathbb{C}^{r}}$ to determine its Euler number in terms of punctual $r$-dimensional partitions of $n$ (the torus actions on the punctual Hilbert scheme $\operatorname{Hilb}^{n}\left(\mathbb{C}^{r}, O\right)$ were studied in [2]). This allows us to prove Theorem 1.2.

It would be interesting to see whether (1.2) is true for $r>3$. In addition, many results and techniques in this paper can be generalized to handle other situations, e.g., when the class $\beta$ is replaced by a higher multiple of the class of a fiber of $\mu$. We plan to discuss these generalizations in a forthcoming paper.

The paper is organized as follows. In Section 2, the basic properties of virtual Hodge polynomials are reviewed. In Section 3 , we identify $\mathfrak{M}_{n}$ with 
certain Grothendieck Quot-scheme and introduce a natural decomposition of $\mathfrak{M}_{n}$. In Section 4, various bijective morphisms are constructed. In Section. 5, we reduce the computation to the local model $\mathbb{C}^{r-1} \times C$. In Section 6 , we further reduce the computation from the local model $\mathbb{C}^{r-1} \times C$ to the punctual spaces $\mathfrak{M}_{n, L, O}^{\mathbb{C}^{r}}$ and $\mathrm{Hilb}^{n}\left(\mathbb{C}^{r}, O\right)$. In Section. 7, we use torus actions to verify Theorem 1.2 and (1.2) when $r=3$.

\section{Virtual Hodge polynomials and Euler numbers.}

Danilov and Khovanskii [3] introduced virtual Hodge polynomials for reduced complex schemes. These polynomials can be viewed as a convenient tool for computing the Hodge numbers of smooth projective varieties by reducing to computing those of simpler varieties. They can also be used to compute Euler numbers. In this section, we recall the basic properties of virtual Hodge polynomials.

First of all, let $Y$ be a reduced complex scheme (not necessarily projective, irreducible or smooth). Mixed Hodge structures are defined on the cohomology $H_{c}^{k}(Y, \mathbb{Q})$ with compact support (see $\left.[4,3]\right)$. The mixed Hodge structures coincide with the classical one if $Y$ is projective and smooth. For each pair of integers $(m, n)$, define the virtual Hodge number

$$
e^{m, n}(Y)=\sum_{k}(-1)^{k} h^{m, n}\left(H_{c}^{k}(Y, \mathbb{Q})\right) .
$$

Then the virtual Hodge polynomial of $Y$ is defined to be

$$
e(Y ; s, t)=\sum_{m, n} e^{m, n}(Y) s^{m} t^{n}
$$

Next, for an arbitrary complex scheme $Y$, we put

$$
e(Y ; s, t)=e\left(Y_{\text {red }} ; s, t\right)
$$

following [2]. By (2.2) and the results in [3, 6, 2] for reduced complex schemes, we see that virtual Hodge polynomials satisfy the following properties:

(i) When $Y$ is projective and smooth, $e(Y ; s, t)$ is the usual Hodge polynomial of $Y$. For a general complex scheme $Y$, we have

$$
e(Y ; 1,1)=\chi(Y)
$$

where $\chi(Y)$ denotes the topological Euler number of $Y$. 
(ii) If $Y=\coprod_{i=1}^{n} Y_{i}$ is a finite disjoint union of locally closed subsets, then

$$
e(Y ; s, t)=\sum_{i=1}^{n} e\left(Y_{i} ; s, t\right)
$$

(iii) If $f: Y \rightarrow Y^{\prime}$ is a Zariski-locally trivial bundle with fiber $F$, then

$$
e(Y ; s, t)=e\left(Y^{\prime} ; s, t\right) \cdot e(F ; s, t)
$$

(iv) If $f: Y \rightarrow Y^{\prime}$ is a bijective morphism, then

$$
e(Y ; s, t)=e\left(Y^{\prime} ; s, t\right)
$$

By the Lemma 5.6 in [2] and the Theorem 4.1 in [12], if $Y$ is a reduced complex scheme with a $\mathbb{C}^{*}$-action and if $Y^{\mathbb{C}^{*}}$ denotes the set of fixed points, then

$$
\chi(Y)=\chi\left(Y^{\mathbb{C}^{*}}\right) .
$$

\section{The moduli spaces $\mathfrak{I}_{n}(X, \beta)$ and $\mathfrak{M}_{n}$.}

Let $X$ be a smooth projective complex variety of dimension $r$. For a fixed class $\beta \in H_{2}(X ; \mathbb{Z})$ and a fixed integer $n$, following the definitions and notations in $[13,14]$, we define $\mathfrak{I}_{n}(X, \beta)$ to be the moduli space of 1 -dimensional closed subschemes $Z$ of $X$ satisfying the two conditions:

$$
\chi\left(\mathcal{O}_{Z}\right)=n, \quad[Z]=\beta
$$

where $[Z]$ is the class associated to the dimension- 1 component (weighted by their intrinsic multiplicities) of $Z$. The degree- 0 moduli space $\mathfrak{I}_{n}(X, 0)$ is isomorphic to the Hilbert scheme $X^{[n]}$ parametrizing length- $n$ 0-dimensional closed subschemes of $X$. In general, when $\beta \neq 0$, the space $\mathfrak{I}_{n}(X, \beta)$ is only part of the Hilbert scheme defined in terms of certain degree-1 Hilbert polynomial (see [9]). By the Lemma 1 in [14], when $\operatorname{dim}(X)=3$, the virtual dimension of $\mathfrak{I}_{n}(X, \beta)$ is

$$
-\left(\beta \cdot K_{X}\right)
$$

In the rest of the paper, we adopt the following basic assumptions. 
Assumption 3.1. We assume that $X$ admits a Zariski-locally trivial fibration

$$
\mu: X \rightarrow S
$$

where $S$ is smooth, the fibers are smooth irreducible curves of genus- $g$, and $\beta \in H_{2}(X ; \mathbb{Z})$ is the class of a fiber. Then, $\mathfrak{I}_{(1-g)}(X, \beta) \cong S$. For $n \geq 0$, let

$$
\mathfrak{M}_{n}:=\mathfrak{I}_{(1-g)+n}(X, \beta) \text {. }
$$

Our goal is to determine, under Assumption 3.1, the partition function for the Euler numbers of the moduli spaces $\mathfrak{M}_{n}=\mathfrak{I}_{(1-g)+n}(X, \beta), n \geq 0$ :

$$
\sum_{n=0}^{+\infty} \chi\left(\mathfrak{M}_{n}\right) q^{n}
$$

Lemma 3.2. Let $\Delta$ be the diagonal of $S \times S$, and $\operatorname{Quot}_{\left(\operatorname{Id}_{S} \times \mu\right)^{*} I_{\Delta} / S \times X / S}^{n}$ be the Grothendieck Quot-scheme with the constant polynomial n. Then, there exists an isomorphism between $\mathfrak{M}_{n}$ and $\operatorname{Quot}_{\left(\operatorname{Id}_{S} \times \mu\right)^{*} I_{\Delta} / S \times X / S}^{n}$.

Proof. Note that every element in $\mathfrak{M}_{n}=\mathfrak{I}_{(1-g)+n}(X, \beta)$ is of the form:

$$
Z=\Xi+\Theta
$$

where $\Xi \in X^{\left[n-n_{0}\right]}$ for some $n_{0}$ satisfying $0 \leq n_{0} \leq n, \operatorname{Supp}(\Xi) \cap \operatorname{Supp}(\Theta)=$ $\emptyset$, and the dimension- 1 component $\Theta$ is equal to some curve $C \in \mathfrak{M}_{0}$ together with embedded points of length- $n_{0}$ (i.e., $I_{\Theta} \subset I_{C}$ and the quotient $I_{C} / I_{\Theta}$ is supported at finitely many points in $C$ with $\left.h^{0}\left(X, I_{C} / I_{\Theta}\right)=n_{0}\right)$. So we have a surjection

$$
I_{C} \rightarrow I_{C} / I_{Z} \rightarrow 0
$$

where the quotient $I_{C} / I_{Z}$ is supported at finitely many points, and has length $n$.

It follows that the universal quotient over $\operatorname{Quot}_{\left(\operatorname{Id}_{S} \times \mu\right)^{*} I_{\Delta} / S \times X / S}^{n} \times X$ induces a bijective morphism $\phi_{1}:$ Quot $_{\left(\operatorname{Id}_{S} \times \mu\right)^{*} I_{\Delta} / S \times X / S}^{n} \rightarrow \mathfrak{M}_{n}$.

On the other hand, let $\mathcal{I}_{n}$ be the universal ideal sheaf over $\mathfrak{M}_{n} \times X$. Let $\mathcal{I}_{n}^{\prime}$ be the saturation of $\mathcal{I}_{n} \subset \mathcal{O}_{\mathfrak{M}_{n} \times X}$ (see Definition 1.1.5 in [10]). Then, $\mathcal{I}_{n}^{\prime}$ is a flat family of ideal sheaves in $\mathfrak{M}_{0} \cong S$, and fits in an exact sequence

$$
0 \rightarrow \mathcal{I}_{n} \rightarrow \mathcal{I}_{n}^{\prime} \rightarrow \mathcal{Q} \rightarrow 0
$$


over $\mathfrak{M}_{n} \times X$. Now the flat family $\mathcal{I}_{n}^{\prime}$ and the quotient $\mathcal{I}_{n}^{\prime} \rightarrow \mathcal{Q} \rightarrow 0$ induces a morphism $\phi_{2}: \mathfrak{M}_{n} \rightarrow \operatorname{Quot}_{\left(\operatorname{Id}_{S} \times \mu\right)^{*} I_{\Delta} / S \times X / S}^{n}$ which is inverse to $\phi_{1}$.

In view of Lemma 3.2 , we will make no difference between $\mathfrak{M}_{n}$ and

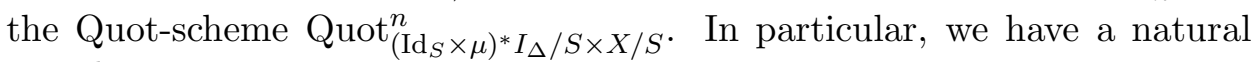
morphism:

$$
\mathfrak{M}_{n} \rightarrow S
$$

Moreover, over $\mathfrak{M}_{n} \times X$, there exists a universal quotient

$$
p^{*} I_{\Delta} \rightarrow \mathcal{Q} \rightarrow 0
$$

where $p$ is the composition of the morphism $\mathfrak{M}_{n} \times X \rightarrow S \times X$ induced from (3.7) and the morphism $\operatorname{Id}_{S} \times \mu: S \times X \rightarrow S \times S$.

Definition 3.3. Let $\beta$ and $g$ be from Assumption 3.1. Let $0 \leq n_{0} \leq n$.

(i) We define $\mathfrak{M}_{n, n_{0}}$ to be the locally closed subset of $\mathfrak{M}_{n}$ consisting of all the elements $Z=\Xi+\Theta$ from (3.6) such that $h^{0}\left(X, I_{C} / I_{\Theta}\right)=n_{0}$.

(ii) Fix a fiber $C$ of $\mu$ and a point $x \in C$. We define $\mathfrak{M}_{n, C}$ (respectively, $\left.\mathfrak{M}_{n, C, x}\right)$ to be the closed subset of $\mathfrak{M}_{n, n}$ consisting of all the elements $Z=\Xi+\Theta$ from (3.6) such that $\Xi=\emptyset, I_{\Theta} \subset I_{C}$, and $I_{C} / I_{\Theta}$ is supported at finitely many points (respectively, supported at the point $x$ ).

(iii) Fix a fiber $C$ of $\mu$, and a point $x \in X$. Define $X_{C}^{[n], n_{0}}$ to be the locally closed subset of $X^{[n]}$ consisting of all the elements $\Xi=\Xi_{1}+\Xi_{2} \in X^{[n]}$ such that $\operatorname{Supp}\left(\Xi_{1}\right) \cap C=\emptyset, \operatorname{Supp}\left(\Xi_{2}\right) \subset C$, and $\ell\left(\Xi_{2}\right)=n_{0}$. Define $X_{C}^{[n]}=X_{C}^{[n], n}$, and define $X_{x}^{[n]}$ to be the closed subset of $X^{[n]}$ consisting of all $\Xi \in X^{[n]}$ such that $\operatorname{Supp}(\Xi)=\{x\}$ (i.e., $X_{x}^{[n]}$ is the punctual Hilbert scheme at $x$ ).

Remark 3.4. To emphasize the dependence on $X$, we will also denote the notations $\mathfrak{M}_{n}, \mathfrak{M}_{n, n_{0}}, \mathfrak{M}_{n, C}, \ldots$ by $\mathfrak{M}_{n}^{X}, \mathfrak{M}_{n, n_{0}}^{X}, \mathfrak{M}_{n, C}^{X}, \ldots$ respectively.

\section{Various bijective morphisms.}

We begin with an outline of this section. Consider the variety $X$ as in Assumption 3.1. By Definition 3.3 (i), $\mathfrak{M}_{n}$ has a decomposition of locally closed subsets:

$$
\mathfrak{M}_{n}=\coprod_{n_{0}=0}^{n} \mathfrak{M}_{n, n_{0}}
$$


In order to use the method of virtual Hodge polynomials, we need that there is a Zariski-locally trivial fibration space mapping to $\mathfrak{M}_{n, n_{0}}$ bijectively. For instance, we see from (3.7) that $\mathfrak{M}_{n, n}$ admits a morphism to $S$. The fiber of this map over $s \in S$ is isomorphic to $\mathfrak{M}_{n, C}$ where $C=\mu^{-1}(s)$ is a fiber of $\mu$ : $X \rightarrow S$. If we consider another fiber $C^{\prime}=\mu^{-1}\left(s^{\prime}\right)$, then $\mathfrak{M}_{n, C}$ is isomorphic to $\mathfrak{M}_{n, C^{\prime}}$. In view of $(2.5)$ and (2.6), it suffices to prove that $\mathfrak{M}_{n, C} \times S$ admits a bijective morphism to $\mathfrak{M}_{n, n}$ locally over $S$. To achieve this, we first consider the local model where we take $X=\mathbb{C}^{m} \times C$ in Lemma 4.1. For the general case $\mu: X \rightarrow S$, we choose an open subset $U$ of $S$ such that there exists an étale morphism $U \rightarrow \mathbb{C}^{m}$. Then we prove in Lemma 4.2 the existence of a bijective morphism $\mathfrak{M}_{n, C}^{\mathbb{C}^{m} \times C} \times_{\mathbb{C}^{m}} U \rightarrow \mathfrak{M}_{n, n}^{U \times C}$. For other strata $\mathfrak{M}_{n, n_{0}}$, Lemma 4.6 provides a kind of locally trivial fibration description.

Now we fix some notations. Let $C$ denote a fixed fiber of the fibration $\mu: X \rightarrow S$. Let $m=\operatorname{dim}(X)-1=r-1, O$ be the origin of $\mathbb{C}^{m}$, and $C_{0}=\{O\} \times C$.

Lemma 4.1. There exists a bijective morphism over $\mathbb{C}^{m}$ :

$$
\Psi: \mathfrak{M}_{n, C_{0}}^{\mathbb{C}^{m} \times C} \times \mathbb{C}^{m} \rightarrow \mathfrak{M}_{n, n}^{\mathbb{C}^{m} \times C}
$$

Proof. Let $X_{0}=\mathbb{C}^{m} \times C$. Over $\mathfrak{M}_{n, C_{0}}^{X_{0}} \times X_{0}$, there exists an universal quotient

$$
p_{2}^{*} I_{C_{0}} \rightarrow \mathcal{Q}_{0} \rightarrow 0
$$

where $p_{2}: \mathfrak{M}_{n, C_{0}}^{X_{0}} \times X_{0} \rightarrow X_{0}$ is the second projection. Let

$$
\sigma: \mathbb{C}^{m} \times \mathbb{C}^{m} \rightarrow \mathbb{C}^{m}
$$

be the subtraction: $\sigma(u, v)=u-v$. Let $\Sigma=\operatorname{Id}_{\mathfrak{M}_{n, C_{0}}^{X_{0}}} \times \sigma \times \operatorname{Id}_{C}$ :

$$
\mathfrak{M}_{n, C_{0}}^{X_{0}} \times \mathbb{C}^{m} \times X_{0}=\mathfrak{M}_{n, C_{0}}^{X_{0}} \times \mathbb{C}^{m} \times \mathbb{C}^{m} \times C \rightarrow \mathfrak{M}_{n, C_{0}}^{X_{0}} \times \mathbb{C}^{m} \times C=\mathfrak{M}_{n, C_{0}}^{X_{0}} \times X_{0}
$$

Then we obtain a commutation diagram of morphisms:

$$
\begin{array}{cccc}
\mathfrak{M}_{n, C_{0}}^{X_{0}} \times \mathbb{C}^{m} \times X_{0} & \stackrel{\Sigma}{\rightarrow} & \mathfrak{M}_{n, C_{0}}^{X_{0}} \times X_{0} \\
\downarrow & & \downarrow \\
\mathbb{C}^{m} \times \mathbb{C}^{m} & \stackrel{\sigma}{\rightarrow} & \mathbb{C}^{m}
\end{array}
$$

where the two vertical morphisms are the natural projections. We have

$$
\Sigma^{*}\left(p_{2}^{*} I_{C_{0}}\right) \rightarrow \Sigma^{*} \mathcal{Q}_{0} \rightarrow 0
$$


over $\mathfrak{M}_{n, C_{0}}^{X_{0}} \times \mathbb{C}^{m} \times X_{0}$. Let $\Delta_{0}$ be the diagonal of $\mathbb{C}^{m} \times \mathbb{C}^{m}$. Then, we see that $\Sigma^{*}\left(p_{2}^{*} I_{C_{0}}\right)=I_{\pi^{-1} \Delta_{0}}$. Therefore, over $\mathfrak{M}_{n, C_{0}}^{X_{0}} \times \mathbb{C}^{m} \times X_{0}$, we have

$$
I_{\pi^{-1} \Delta_{0}} \rightarrow \Sigma^{*} \mathcal{Q}_{0} \rightarrow 0 .
$$

Let $u \in \mathbb{C}^{m}$. The restriction of (4.3) to $\mathfrak{M}_{n, C_{0}}^{X_{0}} \times\{u\} \times X_{0} \cong \mathfrak{M}_{n, C_{0}}^{X_{0}} \times X_{0}$ is

$$
p_{2}^{*} I_{\{u\} \times C}=\Sigma_{u}^{*}\left(p_{2}^{*} I_{C_{0}}\right) \rightarrow \Sigma_{u}^{*} \mathcal{Q}_{0} \rightarrow 0
$$

where $\Sigma_{u}$ is the automorphism of $\mathfrak{M}_{n, C_{0}}^{X_{0}} \times X_{0}=\mathfrak{M}_{n, C_{0}}^{X_{0}} \times \mathbb{C}^{m} \times C$ induced by

$$
\sigma_{u}: \mathbb{C}^{m} \rightarrow \mathbb{C}^{m}
$$

with $\sigma_{u}(v)=u-v$. By the universal property, (4.3) induces a morphism:

$$
\Psi: \mathfrak{M}_{n, C_{0}}^{X_{0}} \times \mathbb{C}^{m} \rightarrow \mathfrak{M}_{n, n}^{X_{0}}
$$

The morphism $\Psi$ is bijective since every $\Sigma_{u}$ is an automorphism.

Lemma 4.2. Let $f: U \rightarrow \mathbb{C}^{m}$ be an étale morphism. Then there exists a bijective morphism $\widetilde{\Psi}_{f}: \mathfrak{M}_{n, n}^{\mathbb{C}} \times C \times \times_{\mathbb{C}^{m}} U \rightarrow \mathfrak{M}_{n, n}^{U \times C}$ over $U$.

Proof. Let $X=U \times C$ and $X_{0}=\mathbb{C}^{m} \times C$. Then there exists a universal quotient

$$
\left(\pi_{0}\right)^{*} I_{\Delta_{0}} \rightarrow \mathcal{Q} \rightarrow 0
$$

over $\mathfrak{M}_{n, n}^{X_{0}} \times X_{0}$, where $\Delta_{0}$ is the diagonal of $\mathbb{C}^{m} \times \mathbb{C}^{m}$ and $\pi_{0}$ is the composition:

$$
\mathfrak{M}_{n, n}^{X_{0}} \times X_{0} \rightarrow \mathbb{C}^{m} \times X_{0}=\mathbb{C}^{m} \times\left(\mathbb{C}^{m} \times C\right) \rightarrow \mathbb{C}^{m} \times \mathbb{C}^{m} .
$$

The projection $\mathfrak{M}_{n, n}^{X_{0}} \times \mathbb{C}^{m} U \rightarrow \mathfrak{M}_{n, n}^{X_{0}}$ and the morphism $f \times \operatorname{Id}_{C}: X \rightarrow X_{0}$ induces

$$
F:\left(\mathfrak{M}_{n, n}^{X_{0}} \times \mathbb{C}^{m} U\right) \times X \rightarrow \mathfrak{M}_{n, n}^{X_{0}} \times X_{0}
$$

which can also be regarded as the base change of $f \times f: U \times U \rightarrow \mathbb{C}^{m} \times \mathbb{C}^{m}$ by $\pi_{0}$ :

$$
\begin{array}{rlrl}
\left(\mathfrak{M}_{n, n}^{X_{0}} \times \mathbb{C}^{m} U\right) \times X & \longrightarrow & U & \times U \\
\downarrow F & & & \downarrow f \times f \\
\mathfrak{M}_{n, n}^{X_{0}} \times X_{0} & \stackrel{\pi_{0}}{\longrightarrow} & \mathbb{C}^{m} \times \mathbb{C}^{m} .
\end{array}
$$


Pulling-back the surjection (4.4) via $F$, we obtain the surjection

$$
F^{*}\left(\pi_{0}\right)^{*} I_{\Delta_{0}} \rightarrow F^{*} \mathcal{Q} \rightarrow 0
$$

over $\left(\mathfrak{M}_{n, n}^{X_{0}} \times \mathbb{C}^{m} U\right) \times X$. Regard $U \times U$ as a scheme over $\mathbb{C}^{m}$ by using

$$
U \times U \stackrel{p_{1}}{\rightarrow} U \stackrel{f}{\rightarrow} \mathbb{C}^{m}
$$

where $p_{1}$ is the first projection of $U \times U$. Then,

$$
\left(\mathfrak{M}_{n, n}^{X_{0}} \times \mathbb{C}^{m} U\right) \times X=\mathfrak{M}_{n, n}^{X_{0}} \times_{\mathbb{C}^{m}}(U \times U) \times C .
$$

Let $\Delta$ be the diagonal of $U \times U$, and $\pi$ be the composition:

$$
\left(\mathfrak{M}_{n, n}^{X_{0}} \times_{\mathbb{C}^{m}} U\right) \times X=\mathfrak{M}_{n, n}^{X_{0}} \times_{\mathbb{C}^{m}}(U \times U) \times C \rightarrow U \times U
$$

Restricting (4.6) to $\pi^{-1}(\Delta) \subset\left(\mathfrak{M}_{n, n}^{X_{0}} \times_{\mathbb{C}^{m}} U\right) \times X$, we obtain

$$
\left.\left.F^{*}\left(\pi_{0}\right)^{*} I_{\Delta_{0}}\right|_{\pi^{-1}(\Delta)} \rightarrow F^{*} \mathcal{Q}\right|_{\pi^{-1}(\Delta)} \rightarrow 0 .
$$

Note that $\pi^{-1}(\Delta) \subset\left(\pi_{0} \circ F\right)^{-1}\left(\Delta_{0}\right)$. In fact, since $f$ is étale, $\left(\pi_{0} \circ F\right)^{-1}\left(\Delta_{0}\right)$ is the disjoint union of $\pi^{-1}(\Delta)$ and some other irreducible components. Hence

$\left.F^{*}\left(\pi_{0}\right)^{*} I_{\Delta_{0}}\right|_{\pi^{-1}(\Delta)}=\left.I_{\left(\pi_{0} \circ F\right)^{-1}\left(\Delta_{0}\right)}\right|_{\pi^{-1}(\Delta)}=\left.I_{\pi^{-1}(\Delta)}\right|_{\pi^{-1}(\Delta)}=\left.\pi^{*} I_{\Delta}\right|_{\pi^{-1}(\Delta)}$.

Using (4.8) and the surjection $\left.\pi^{*} I_{\Delta} \rightarrow \pi^{*} I_{\Delta}\right|_{\pi^{-1}(\Delta)}$, we obtain

$$
\left.\pi^{*} I_{\Delta} \rightarrow F^{*} \mathcal{Q}\right|_{\pi^{-1}(\Delta)} \rightarrow 0
$$

over $\left(\mathfrak{M}_{n, n}^{X_{0}} \times \mathbb{C}^{m} U\right) \times X$. One checks that $\left.F^{*} \mathcal{Q}\right|_{\pi^{-1}(\Delta)}$ is flat over $\mathfrak{M}_{n, n}^{X_{0}} \times \mathbb{C}^{m} U$ and that the quotient (4.9) induces a morphism:

$$
\widetilde{\Psi}_{f}: \mathfrak{M}_{n, n}^{X_{0}} \times_{\mathbb{C}^{m}} U \rightarrow \mathfrak{M}_{n, n}^{X}
$$

over $U$. Using the completions of the points in $U$, we see that $\widetilde{\Psi}_{f}$ is bijective.

Proposition 4.3. Let $O$ be the origin of $\mathbb{C}^{m}$ and $C_{0}=\{O\} \times C$. Let $f: U \rightarrow \mathbb{C}^{m}$ be an étale morphism. Then there exists a bijective morphism over $U$ :

$$
\Psi_{f}: \mathfrak{M}_{n, C_{0}}^{\mathbb{C}^{m} \times C} \times U \rightarrow \mathfrak{M}_{n, n}^{U \times C}
$$


Proof. Follows from Lemmas 4.1 and 4.2 by putting $\Psi_{f}=\widetilde{\Psi}_{f} \circ\left(\Psi \times_{\mathbb{C}^{m}}\right.$ $\left.\operatorname{Id}_{U}\right)$.

Remark 4.4. The bijective morphism $\Psi_{f}$ in Proposition 4.3 is in fact an isomorphism. To see this, we use an analytic open covering of $U$ to show that $\Psi_{f}$ is an isomorphism locally in analytic category. This coupled with the bijectivity implies that the morphism $\Psi_{f}$ is indeed an isomorphism.

Definition 4.5. For $0 \leq n_{0} \leq n$, define $Z_{n, i}$ to be the locally closed subset of $X^{[n]} \times S$ consisting of all the pairs $(\Xi, s)$ such that $\Xi=\Xi_{1}+\Xi_{2}$ with $\operatorname{Supp}\left(\Xi_{1}\right) \cap \mu^{-1}(s)=\emptyset, \operatorname{Supp}\left(\Xi_{2}\right) \subset \mu^{-1}(s)$, and $\ell\left(\Xi_{1}\right)=i$. Put

$$
W_{n}=Z_{n, n}, \quad T_{n}=Z_{n, 0} .
$$

Note that we have natural morphisms $Z_{n, i} \rightarrow X^{[n]}$ and $Z_{n, i} \rightarrow S$.

Lemma 4.6. Let $0 \leq n_{0} \leq n$. Then there exists a bijective morphism:

$$
W_{n-n_{0}} \times_{S} \mathfrak{M}_{n_{0}, n_{0}} \rightarrow \mathfrak{M}_{n, n_{0}} .
$$

Proof. Let $\pi_{1}$ and $\pi_{2}$ be the two natural projections of $W_{n-n_{0}} \times_{S} \mathfrak{M}_{n_{0}, n_{0}}$. We see from (3.8) that over $\mathfrak{M}_{n_{0}, n_{0}} \times X$, there exists an universal quotient

$$
p^{*} I_{\Delta} \rightarrow \mathcal{Q}_{1} \rightarrow 0
$$

So over $\left(W_{n-n_{0}} \times_{S} \mathfrak{M}_{n_{0}, n_{0}}\right) \times X$, we have a surjection:

$$
\left(\pi_{2} \times \operatorname{Id}_{X}\right)^{*} p^{*} I_{\Delta} \rightarrow\left(\pi_{2} \times \operatorname{Id}_{X}\right)^{*} \mathcal{Q}_{1} \rightarrow 0 .
$$

In addition, over $X^{\left[n-n_{0}\right]} \times X$, we have a universal quotient

$$
\mathcal{O}_{X^{\left[n-n_{0}\right]} \times X} \rightarrow \mathcal{Q}_{2} \rightarrow 0
$$

Hence over $\left(W_{n-n_{0}} \times_{S} \mathfrak{M}_{n_{0}, n_{0}}\right) \times X$, we have another surjection:

$$
\mathcal{O}_{\left(W_{n-n_{0}} \times{ }_{S} \mathfrak{M}_{n_{0}, n_{0}}\right) \times X} \rightarrow \pi^{*} \mathcal{Q}_{2} \rightarrow 0
$$

where $\pi$ is the composition of $\pi_{1} \times \operatorname{Id}_{X}:\left(W_{n-n_{0}} \times{ }_{S} \mathfrak{M}_{n_{0}, n_{0}}\right) \times X \rightarrow W_{n-n_{0}} \times$ $X$ and the natural morphism $W_{n-n_{0}} \times X \rightarrow X^{\left[n-n_{0}\right]} \times X$. Note that

$$
\begin{gathered}
\operatorname{Supp}\left(\left(\pi_{2} \times \operatorname{Id}_{X}\right)^{*} \mathcal{Q}_{1}\right) \subset\left(\pi_{2} \times \operatorname{Id}_{X}\right)^{-1} p^{-1}(\Delta), \\
\left(\pi_{2} \times \operatorname{Id}_{X}\right)^{-1} p^{-1}(\Delta) \cap \operatorname{Supp}\left(\pi^{*} \mathcal{Q}_{2}\right)=\emptyset .
\end{gathered}
$$


Hence $\left(\pi_{2} \times \operatorname{Id}_{X}\right)^{*} \mathcal{Q}_{1} \oplus \pi^{*} \mathcal{Q}_{2}$ is flat over $W_{n-n_{0}}{ }_{S} \mathfrak{M}_{n_{0}, n_{0}}$. Moreover, combining the two surjections (4.10) and (4.11), we obtain a surjection:

$$
\left(\pi_{2} \times \operatorname{Id}_{X}\right)^{*} p^{*} I_{\Delta} \rightarrow\left(\pi_{2} \times \operatorname{Id}_{X}\right)^{*} \mathcal{Q}_{1} \oplus \pi^{*} \mathcal{Q}_{2} \rightarrow 0 .
$$

This surjection induces a morphism $\psi: W_{n-n_{0}} \times_{S} \mathfrak{M}_{n_{0}, n_{0}} \rightarrow \mathfrak{M}_{n}$. One checks that $\operatorname{im}(\psi)=\mathfrak{M}_{n, n_{0}}$ and that $\psi: W_{n-n_{0}} \times_{S} \mathfrak{M}_{n_{0}, n_{0}} \rightarrow \mathfrak{M}_{n, n_{0}}$ is injective.

Remark 4.7. A similar argument shows that there exists a bijective morphism:

$$
W_{n-n_{0}} \times_{S} T_{n_{0}} \rightarrow Z_{n, n_{0}} .
$$

\section{Reduction to the local model $\mathbb{C}^{r-1} \times C$.}

In this section, using the results proved in the previous section, we reduce the computation of the virtual Hodge polynomial of $\mathfrak{M}_{n}$ to those of $X^{[n]}$, $\mathfrak{M}_{n, C_{0}}^{\mathbb{C}^{r-1} \times C}$, and $\left(\mathbb{C}^{r-1} \times C\right)_{C_{0}}^{[n]}$ where $C_{0}=\{O\} \times C$ and $O$ is the origin of $\mathbb{C}^{r-1}$.

Lemma 5.1. Let $O$ be the origin of $\mathbb{C}^{r-1}$ and $C_{0}=\{O\} \times C$. Then,

$$
\sum_{n=0}^{+\infty} e\left(\mathfrak{M}_{n} ; s, t\right) q^{n}=\sum_{n=0}^{+\infty} e\left(W_{n} ; s, t\right) q^{n} \cdot \sum_{n=0}^{+\infty} e\left(\mathfrak{M}_{n, C_{0}}^{\mathbb{C}^{r-1} \times C} ; s, t\right) q^{n}
$$

Proof. By (4.1), Lemma 4.6, (2.4) and (2.6), we obtain:

$$
e\left(\mathfrak{M}_{n} ; s, t\right)=\sum_{n_{0}=0}^{n} e\left(\mathfrak{M}_{n, n_{0}} ; s, t\right)=\sum_{n_{0}=0}^{n} e\left(W_{n-n_{0}} \times_{S} \mathfrak{M}_{n_{0}, n_{0}} ; s, t\right) .
$$

Consider the commutative diagram for the fiber product $W_{n-n_{0}} \times_{S} \mathfrak{M}_{n_{0}, n_{0}}$ :

$$
\begin{array}{ccc}
W_{n-n_{0}} \times_{S} \mathfrak{M}_{n_{0}, n_{0}} & \longrightarrow & \mathfrak{M}_{n_{0}, n_{0}} \\
\downarrow \phi_{1} & & \downarrow \phi_{2} \\
W_{n-n_{0}} & \longrightarrow & S .
\end{array}
$$

By the Proposition I.3.24 in [16], there exists an open affine cover $\left\{U_{i}\right\}_{i}$ of $S$ and étale morphisms $f_{i}: U_{i} \rightarrow \mathbb{C}^{r-1}$. By Proposition 4.3, we see that for each $i$, there exists a bijective morphism over the open affine subset $U_{i}$ :

$$
\Psi_{f_{i}}: \mathfrak{M}_{n_{0}, C_{0}}^{\mathbb{C}^{r-1} \times C} \times U_{i} \rightarrow\left(\phi_{2}\right)^{-1}\left(U_{i}\right) .
$$


So there exist a decomposition $S=\coprod_{i} S_{i}$ of locally closed subsets $S_{i}$ and bijective morphisms $\Psi_{S_{i}}: \mathfrak{M}_{n_{0}, C_{0}}^{\mathbb{C}^{r-1} \times C} \times S_{i} \rightarrow\left(\phi_{2}\right)^{-1}\left(S_{i}\right)$. By (5.3), there exists a decomposition

$$
W_{n-n_{0}}=\coprod_{i} W_{n-n_{0}, i}
$$

of locally closed subsets $W_{n-n_{0}, i}$ and bijective morphisms

$$
\Psi_{W_{n-n_{0}, i}}: \mathfrak{M}_{n_{0}, C_{0}}^{\mathbb{C}^{r-1} \times C} \times W_{n-n_{0}, i} \rightarrow\left(\phi_{1}\right)^{-1}\left(W_{n-n_{0}, i}\right) .
$$

Combining this with (2.4) and (2.6), we conclude that

$$
\begin{aligned}
e\left(W_{n-n_{0}} \times{ }_{S} \mathfrak{M}_{n_{0}, n_{0}} ; s, t\right) & =\sum_{i} e\left(\left(\phi_{1}\right)^{-1}\left(W_{n-n_{0}, i}\right) ; s, t\right) \\
& =\sum_{i} e\left(\mathfrak{M}_{n_{0}, C_{0}}^{\mathbb{C}^{r-1} \times C} \times W_{n-n_{0}, i} ; s, t\right) \\
& =\sum_{i} e\left(W_{n-n_{0}, i} ; s, t\right) \cdot e\left(\mathfrak{M}_{n_{0}, C_{0}}^{\mathbb{C}^{r-1} \times C} ; s, t\right) \\
& =e\left(W_{n-n_{0}} ; s, t\right) \cdot e\left(\mathfrak{M}_{n_{0}, C_{0}}^{\mathbb{C}^{r-1} \times C} ; s, t\right) .
\end{aligned}
$$

Now (5.1) follows immediately from (5.2) and (5.4).

Lemma 5.2. Let $O$ be the origin of $\mathbb{C}^{r-1}$ and $C_{0}=\{O\} \times C$. Then,

$$
\sum_{n=0}^{+\infty} e\left(X^{[n]} \times S ; s, t\right) q^{n}=\sum_{n=0}^{+\infty} e\left(W_{n} ; s, t\right) q^{n} \cdot \sum_{n=0}^{+\infty} e\left(\left(\mathbb{C}^{r-1} \times C\right)_{C_{0}}^{[n]} ; s, t\right) q^{n}
$$

Proof. By Remark 4.7, we have an analogue of (5.2):

$$
e\left(X^{[n]} \times S ; s, t\right)=\sum_{n_{0}=0}^{n} e\left(W_{n-n_{0}} \times{ }_{S} T_{n_{0}} ; s, t\right) .
$$

Let $\lambda$ be a partition of $n_{0}$, denoted by $\lambda \vdash n_{0}$. Express $\lambda$ as $\lambda=\left(\lambda_{1}, \ldots, \lambda_{\ell}\right)$ where $\lambda_{1} \geq \ldots \geq \lambda_{\ell}$ and $\lambda_{1}+\ldots+\lambda_{\ell}=n_{0}$. We define $T_{\lambda}$ to be the locally closed subset of $T_{n_{0}}$ consisting of all the pairs $(\Xi, s)$ such that $\Xi=$ $\Xi_{1}+\ldots+\Xi_{\ell}$ where

$$
\operatorname{Supp}\left(\Xi_{i}\right)=\left\{x_{i}\right\} \subset \mu^{-1}(s),
$$


$\ell\left(\Xi_{i}\right)=\lambda_{i}$, and the points $x_{1}, \ldots, x_{\ell}$ are distinct. Then,

$$
e\left(X^{[n]} \times S ; s, t\right)=\sum_{n_{0}=0}^{n} \sum_{\lambda \vdash n_{0}} e\left(W_{n-n_{0}} \times_{S} T_{\lambda} ; s, t\right) .
$$

Using the Lemma 2.1.4 in [8], we can prove that the natural morphism $T_{\lambda} \rightarrow S$ is a Zariski-locally trivial fibration with fibers isomorphic to $\left(\mathbb{C}^{r-1} \times\right.$ $C)_{C_{0}}^{\lambda}$. Here $\left(\mathbb{C}^{r-1} \times C\right)_{C_{0}}^{\lambda}$ denotes the locally closed subset of $\left(\mathbb{C}^{r-1} \times C\right)_{C_{0}}^{\left[n_{0}\right]}$ consisting of

$$
\Xi^{\prime}=\Xi_{1}^{\prime}+\ldots+\Xi_{\ell}^{\prime}
$$

where $\operatorname{Supp}\left(\Xi_{i}^{\prime}\right)=\left\{x_{i}^{\prime}\right\} \subset C_{0}, \ell\left(\Xi_{i}^{\prime}\right)=\lambda_{i}$, and $x_{1}^{\prime}, \ldots, x_{\ell}^{\prime}$ are distinct. Hence

$$
\begin{aligned}
e\left(X^{[n]} \times S ; s, t\right) & =\sum_{n_{0}=0}^{n} \sum_{\lambda \vdash n_{0}} e\left(W_{n-n_{0}} ; s, t\right) \cdot e\left(\left(\mathbb{C}^{r-1} \times C\right)_{C_{0}}^{\lambda} ; s, t\right) \\
& =\sum_{n_{0}=0}^{n} e\left(W_{n-n_{0}} ; s, t\right) \cdot \sum_{\lambda \vdash n_{0}} e\left(\left(\mathbb{C}^{r-1} \times C\right)_{C_{0}}^{\lambda} ; s, t\right) \\
& =\sum_{n_{0}=0}^{n} e\left(W_{n-n_{0}} ; s, t\right) \cdot e\left(\left(\mathbb{C}^{r-1} \times C\right)_{C_{0}}^{\left[n_{0}\right]} ; s, t\right),
\end{aligned}
$$

where we used the fact that $\left(\mathbb{C}^{r-1} \times C\right)_{C_{0}}^{\left[n_{0}\right]}$ is the disjoint union of the locally closed subsets $\left(\mathbb{C}^{r-1} \times C\right)_{C_{0}}^{\lambda}, \lambda \vdash n_{0}$. Now (5.5) follows immediately.

Proposition 5.3. Let $O$ be the origin of $\mathbb{C}^{r-1}$ and $C_{0}=\{O\} \times C$. Then,

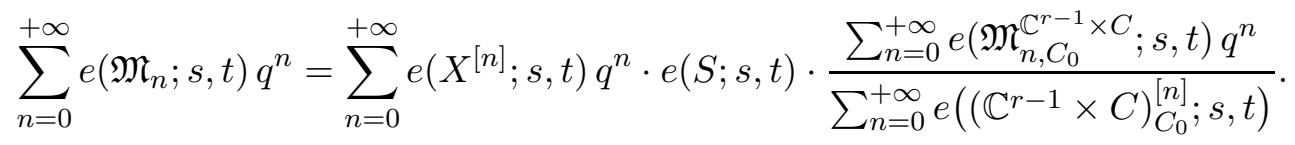

Proof. The formula follows from Lemma 5.1 and Lemma 5.2.

\section{Reduction to the punctual cases.}

From Proposition 5.3, we see that it suffices to compute the virtual Hodge polynomials of $\mathfrak{M}_{n, C_{0}}^{\mathbb{C}^{r-1} \times C}$ and $\left(\mathbb{C}^{r-1} \times C\right)_{C_{0}}^{[n]}$. These spaces are similar to the Hilbert scheme $X^{[n]}$ in the sense that they are all built up from the punctual cases. Cheah developed a method of computing virtual Hodge polynomials to deal with this kind of situation. In order to apply the method to $\mathfrak{M}_{n, C_{0}}^{\mathbb{C}^{r-1} \times C}$ 
and $\left(\mathbb{C}^{r-1} \times C\right)_{C_{0}}^{[n]}$, we first recall Cheah's original approach in [2] for the case of $X^{[n]}$.

Let $\operatorname{Hilb}^{n}\left(\mathbb{C}^{r}, O\right)$ be the punctual Hilbert scheme of $\mathbb{C}^{r}$ at the origin. Then there exist unique rational numbers $H_{\ell, m, n}$ such that

$$
\sum_{n=0}^{+\infty} e\left(\operatorname{Hilb}^{n}\left(\mathbb{C}^{r}, O\right) ; s, t\right) q^{n}=\prod_{\ell=1}^{+\infty} \prod_{m, n=0}^{+\infty}\left(\frac{1}{1-q^{\ell} s^{m} t^{n}}\right)^{H_{\ell, m, n}}
$$

as elements in $\mathbb{Q}[s, t][[q]]$. Define $\mathfrak{h}_{r}(q, s, t) \in \mathbb{Q}[s, t][[q]]$ to be the power series:

$$
\mathfrak{h}_{r}(q, s, t)=\sum_{\ell=1}^{+\infty}\left(\sum_{m, n=0}^{+\infty} H_{\ell, m, n} s^{m} t^{n}\right) q^{\ell}
$$

Then the main result proved in [2] states that

$$
\sum_{n=0}^{+\infty} e\left(X^{[n]} ; s, t\right) q^{n}=\exp \left(\sum_{n=1}^{+\infty} \frac{1}{n} e\left(X ; s^{n}, t^{n}\right) \mathfrak{h}_{r}\left(q^{n}, s^{n}, t^{n}\right)\right) .
$$

The key ingredients in Cheah's proof of (6.3) can be summarized as follows:

(A) Each element $\Xi \in X^{[n]}$ can be uniquely decomposed into $\Xi^{(1)}+\ldots+\Xi^{(\ell)}$ where every $\Xi^{(i)} \in X^{\left[n_{i}\right]}$ is supported at a single point in $X, n_{1}+\ldots+$ $n_{\ell}=n$, and the supports of $\Xi^{(1)}, \ldots, \Xi^{(\ell)}$ are mutually distinct.

(B) Every $X_{x}^{[n]}$ is isomorphic to $\operatorname{Hilb}^{n}\left(\mathbb{C}^{r}, O\right)$. Let $X_{(n)}^{[n]}$ be the closed subscheme of $X^{[n]}$ consisting of all $\Xi \in X^{[n]}$ such that $\operatorname{Supp}(\Xi)$ is a single point of $X$. Then the natural morphism $X_{(n)}^{[n]} \rightarrow X$ sending $\Xi \in$ $X_{(n)}^{[n]}$ to $\operatorname{Supp}(\Xi) \in X$ is Zariski-locally trivial with fibers isomorphic to $\operatorname{Hilb}^{n}\left(\mathbb{C}^{r}, O\right)$.

(C) Using certain combinatorial arguments independent of $X$, one reduces the computation to the virtual Hodge polynomials of $X$ and $\operatorname{Hilb}^{n}\left(\mathbb{C}^{r}, O\right)$ which contribute to the terms $e\left(X ; s^{n}, t^{n}\right)$ and $\mathfrak{h}_{r}\left(q^{n}, s^{n}, t^{n}\right)$ in $(6.3)$ respectively.

It follows that we can apply Cheah's arguments to the computations of

$$
\sum_{n=0}^{+\infty} e\left(\left(\mathbb{C}^{r-1} \times C\right)_{C_{0}}^{[n]} ; s, t\right) q^{n}, \quad \sum_{n=0}^{+\infty} e\left(\mathfrak{M}_{n, C_{0}}^{\mathbb{C}^{r-1} \times C} ; s, t\right) q^{n}
$$


in a straightforward fashion. For $\sum_{n=0}^{+\infty} e\left(\left(\mathbb{C}^{r-1} \times C\right)_{C_{0}}^{[n]} ; s, t\right) q^{n}$, we have

(A1) Each element $\Xi \in\left(\mathbb{C}^{r-1} \times C\right)_{C_{0}}^{[n]}$ can be uniquely decomposed into

$$
\Xi^{(1)}+\ldots+\Xi^{(\ell)}
$$

where each $\Xi^{(i)} \in\left(\mathbb{C}^{r-1} \times C\right)_{C_{0}}^{\left[n_{i}\right]}$ is supported at a single point in $C_{0}$,

$$
n_{1}+\ldots+n_{\ell}=n,
$$

and the supports of $\Xi^{(1)}, \ldots, \Xi^{(\ell)}$ are mutually distinct.

(B1) Every $\left(\mathbb{C}^{r-1} \times C\right)_{x}^{[n]}, x \in C_{0}$ is isomorphic to Hilb ${ }^{n}\left(\mathbb{C}^{r}, O\right)$. The natural morphism $\left(\mathbb{C}^{r-1} \times C\right)_{C_{0}}^{[n]} \rightarrow C_{0}$ sending $\Xi \in\left(\mathbb{C}^{r-1} \times C\right)_{C_{0}}^{[n]}$ to $\operatorname{Supp}(\Xi) \in$ $C_{0}$ is Zariski-locally trivial with fibers isomorphic to $\mathrm{Hilb}^{n}\left(\mathbb{C}^{r}, O\right)$.

(C1) The same combinatorial arguments from (C) reduces the computation to the virtual Hodge polynomials of $C_{0}$ and $\operatorname{Hilb}^{n}\left(\mathbb{C}^{r}, O\right)$.

Therefore, we conclude as in (6.3) the following formula:

$$
\sum_{n=0}^{+\infty} e\left(\left(\mathbb{C}^{r-1} \times C\right)_{C_{0}}^{[n]} ; s, t\right) q^{n}=\exp \left(\sum_{n=1}^{+\infty} \frac{1}{n} e\left(C_{0} ; s^{n}, t^{n}\right) \mathfrak{h}_{r}\left(q^{n}, s^{n}, t^{n}\right)\right) .
$$

Next, for the computation of $\sum_{n=0}^{+\infty} e\left(\mathfrak{M}_{n, C_{0}}^{\mathbb{C}^{r-1} \times C} ; s, t\right) q^{n}$, we have

(A2) Let $\Theta \in \mathfrak{M}_{n, C_{0}}^{\mathbb{C}^{r-1} \times C}$. By the definition of $\mathfrak{M}_{n, C_{0}}^{\mathbb{C}^{r-1} \times C}$, the quotient $I_{C_{0}} / I_{\Theta}$ is supported at finitely many points in $C_{0}$. Put

$$
I_{C_{0}} / I_{\Theta}=Q_{1} \oplus \cdots \oplus Q_{\ell}
$$

where each $Q_{i}$ is supported at a single point in $C_{0}$, and the supports of $Q_{1}, \ldots, Q_{\ell}$ are mutually distinct. Let $f: I_{C_{0}} \rightarrow I_{C_{0}} / I_{\Theta}$ be the quotient map. For $1 \leq i \leq \ell$, define the subscheme $\Theta^{(i)}$ by putting

$$
I_{\Theta^{(i)}}=f^{-1}\left(Q_{i}\right) .
$$

Then $\Theta \in \mathfrak{M}_{n, C_{0}}^{\mathbb{C}^{r-1} \times C}$ gives rise to $\Theta^{(1)}, \ldots, \Theta^{(\ell)}$. It is clear that the process can be reversed. Hence $\Theta \in \mathfrak{M}_{n, C_{0}}^{\mathbb{C}^{r-1} \times C}$ can be formally written as

$$
\Theta=\Theta^{(1)}+\ldots+\Theta^{(\ell)}
$$


in a unique way, where $\Theta^{(i)} \in \mathfrak{M}_{n_{i}, C_{0}}^{\mathbb{C}^{r-1} \times C}$ for $1 \leq i \leq \ell, n_{1}+\ldots+n_{\ell}=n$, each quotient $I_{C_{0}} / I_{\Theta_{i}}$ is supported at a single point in $C_{0}$, and the supports of the quotients $I_{C_{0}} / I_{\Theta_{1}}, \ldots, I_{C_{0}} / I_{\Theta_{\ell}}$ are mutually distinct.

(B2) Let $x \in C_{0}$. Since $C$ is a smooth curve in $X$, we have an isomorphism

$$
\mathfrak{M}_{n, C_{0}, x}^{\mathbb{C}^{r-1} \times C} \cong \mathfrak{M}_{n, L, O}^{\mathbb{C}^{r}}
$$

between the punctual moduli spaces, where $L$ is a coordinate line in $\mathbb{C}^{r}$, $O$ is the origin of $\mathbb{C}^{r}$, and $\mathfrak{M}_{n, L, O}^{\mathbb{C}^{r}}$ parametrizes all the 1-dimensional closed subschemes $\Theta$ of $\mathbb{C}^{r}$ such that $I_{\Theta} \subset I_{L}, \operatorname{Supp}\left(I_{L} / I_{\Theta}\right)=\{O\}$, and

$$
h^{0}\left(\mathbb{C}^{r}, I_{L} / I_{\Theta}\right)=n .
$$

Let $\mathfrak{M}_{(n), C_{0}}^{\mathbb{C}^{r-1} \times C}$ be the subset of $\mathfrak{M}_{n, C_{0}}^{\mathbb{C}^{r-1} \times C}$ consisting of all $\Theta \in \mathfrak{M}_{n, C_{0}}^{\mathbb{C}^{r-1} \times C}$ such that $\operatorname{Supp}\left(I_{C_{0}} / I_{\Theta}\right)$ is a single point in $C_{0}$. By the construction in [9], there is a natural morphism from $\mathfrak{M}_{n}^{\mathbb{C}^{r-1} \times C}$ to the $n$-th symmetric product $\operatorname{Sym}^{n}\left(\mathbb{C}^{r-1} \times C\right)$. Its restriction to $\mathfrak{M}_{(n), C_{0}}^{\mathbb{C}^{r-1} \times C}$ gives rise to a morphism

$$
\phi: \mathfrak{M}_{(n), C_{0}}^{\mathbb{C}^{r-1} \times C} \rightarrow C_{0} .
$$

An argument similar to the proof of Proposition 4.3 shows that there exists a decomposition of locally closed subsets

$$
C_{0}=\coprod_{i} C_{0, i}
$$

and bijective morphisms over the locally closed subsets $C_{0, i}$ :

$$
\Phi_{i}: \mathfrak{M}_{n, L, O}^{\mathbb{C}^{r}} \times C_{0, i} \rightarrow \phi^{-1}\left(C_{0, i}\right) .
$$

(C2) The same combinatorial arguments from (C) reduce the computation to the virtual Hodge polynomials of $C_{0}$ and $\mathfrak{M}_{n, L, O}^{\mathbb{C}^{r}}$.

Hence once again, we conclude as in (6.3) the following:

$$
\sum_{n=0}^{+\infty} e\left(\mathfrak{M}_{n, C_{0}, x}^{\mathbb{C}^{r-1} \times C} ; s, t\right) q^{n}=\exp \left(\sum_{n=1}^{+\infty} \frac{1}{n} e\left(C_{0} ; s^{n}, t^{n}\right) \mathfrak{c}_{r}\left(q^{n}, s^{n}, t^{n}\right)\right)
$$

where the power series $\mathfrak{c}_{r}(q, s, t) \in \mathbb{Q}[s, t][[q]]$ is defined by

$$
\mathfrak{c}_{r}(q, s, t)=\sum_{\ell=1}^{+\infty}\left(\sum_{m, n=0}^{+\infty} C_{\ell, m, n} s^{m} t^{n}\right) q^{\ell}
$$


and the rational numbers $C_{\ell, m, n}$ are the unique rational numbers such that

$$
\sum_{n=0}^{+\infty} e\left(\mathfrak{M}_{n, L, O}^{\mathbb{C}^{r}} ; s, t\right) q^{n}=\prod_{\ell=1}^{+\infty} \prod_{m, n=0}^{+\infty}\left(\frac{1}{1-q^{\ell} s^{m} t^{n}}\right)^{C_{\ell, m, n}}
$$

Lemma 6.1. Let $O$ be the origin of $\mathbb{C}^{r-1}$ and $C_{0}=\{O\} \times C$. Let $\mathfrak{h}_{r}(q, s, t)$ and $\mathfrak{c}_{r}(q, s, t)$ be from (6.2) and (6.7). Then, $\sum_{n=0}^{+\infty} e\left(\mathfrak{M}_{n} ; s, t\right) q^{n}$ is equal to

$$
\begin{gathered}
\sum_{n=0}^{+\infty} e\left(X^{[n]} ; s, t\right) q^{n} \cdot e(S ; s, t) \\
\cdot \exp \left(\sum_{n=1}^{+\infty} \frac{1}{n} e\left(C_{0} ; s^{n}, t^{n}\right)\left[\mathfrak{c}_{r}\left(q^{n}, s^{n}, t^{n}\right)-\mathfrak{h}_{r}\left(q^{n}, s^{n}, t^{n}\right)\right]\right) .
\end{gathered}
$$

Proof. Follows immediately from Lemma 5.3, (6.4) and (6.6).

Proposition 6.2. Under Assumption 3.1, we have

$$
\sum_{n=0}^{+\infty} \chi\left(\mathfrak{M}_{n}\right) q^{n}=\sum_{n=0}^{+\infty} \chi\left(X^{[n]}\right) q^{n} \cdot \chi(S) \cdot\left(\frac{\sum_{n=0}^{+\infty} \chi\left(\mathfrak{M}_{n, L, O}^{\mathbb{C}^{r}}\right) q^{n}}{\sum_{n=0}^{+\infty} \chi\left(\operatorname{Hilb}^{n}\left(\mathbb{C}^{r}, O\right)\right) q^{n}}\right)^{2-2 g}
$$

Proof. Follows from Lemma 6.1, (2.3) and the observation that

$$
\exp \left(\sum_{n=1}^{+\infty} \frac{1}{n} \mathfrak{c}_{r}\left(q^{n}, 1,1\right)\right)=\sum_{n=0}^{+\infty} \chi\left(\mathfrak{M}_{n, L, O}^{\mathbb{C}^{r}}\right) q^{n}
$$

\section{Torus actions on $\operatorname{Hilb}^{n}\left(\mathbb{C}^{r}, O\right)$ and $\mathfrak{M}_{n, L, O}^{\mathbb{C}^{r}}$.}

The last step in our computation is to determine the Euler number of $\mathfrak{M}_{n, L, O}^{\mathbb{C}^{r}}$ (the Euler number of $\operatorname{Hilb}^{n}\left(\mathbb{C}^{r}, O\right)$ has been calculated in [2]). According to (2.7), we can make use of a suitable $\mathbb{C}^{*}$-action on $\mathbb{C}^{r}$ and compute the Euler number of the fixed locus of the induced $\mathbb{C}^{*}$-action on $\mathfrak{M}_{n, L, O}^{\mathbb{C}^{r}}$.

The fixed points of torus actions on the spaces $\operatorname{Hilb}^{n}\left(\mathbb{C}^{r}, O\right)$ and $\mathfrak{M}_{n, L, O}^{\mathbb{C}^{r}}$ are closely related to multi-dimensional partitions of $n$. 
Definition 7.1. (i) Let $r \geq 2$ and $n \geq 0$. An $r$-dimensional partition (respectively, a punctual $r$-dimensional partition) of $n$ is an array

$$
\left(n_{i_{1}, \ldots, i_{r-1}}\right)_{i_{1}, \ldots, i_{r-1}}
$$

of non-negative integers $n_{i_{1}, \ldots, i_{r-1}}$ indexed by the tuples

$$
\left(i_{1}, \ldots, i_{r-1}\right) \in\left(\mathbb{Z}_{\geq 0}\right)^{r-1}
$$

(respectively, by the tuples $\left.\left(i_{1}, \ldots, i_{r-1}\right) \in\left(\mathbb{Z}_{\geq 0}\right)^{r-1}-\{(0, \ldots, 0)\}\right)$ such that

$$
\sum_{i_{1}, \ldots, i_{r-1}} n_{i_{1}, \ldots, i_{r-1}}=n
$$

and $n_{i_{1}, \ldots, i_{r-1}} \geq n_{j_{1}, \ldots, j_{r-1}}$ whenever $i_{1} \leq j_{1}, \ldots, i_{r-1} \leq j_{r-1}$.

(ii) We define $P_{r}(n)$ (respectively, $\widetilde{P}_{r}(n)$ ) to be the number of $r$ dimensional (respectively, punctual $r$-dimensional) partitions of $n$.

We remark that Definition 7.1 (i) is consistent with the one used in [13], while our $r$-dimensional partitions are $(r-1)$-dimensional partitions in [2].

Torus actions on the punctual Hilbert scheme $\operatorname{Hilb}^{n}\left(\mathbb{C}^{r}, O\right)$ have been studied in [2]. Let $z_{1}, \ldots, z_{r}$ be the coordinate functions of $\mathbb{C}^{r}$. Then $\mathbb{C}^{*}$ acts on $\mathbb{C}^{r}$ by

$$
t\left(z_{1}, \ldots, z_{r}\right)=\left(t^{w_{1}} z_{1}, \ldots, t^{w_{r}} z_{r}\right), \quad t \in \mathbb{C}^{*} .
$$

This $\mathbb{C}^{*}$-action on $\mathbb{C}^{r}$ induces a $\mathbb{C}^{*}$-action on $\operatorname{Hilb}^{n}\left(\mathbb{C}^{r}, O\right)$. Now choose $w_{1}, \ldots, w_{r} \in \mathbb{Z}$ properly. Then the $\mathbb{C}^{*}$-fixed points in $\operatorname{Hilb}^{n}\left(\mathbb{C}^{r}, O\right)$ are precisely those corresponding to the colength- $n$ ideals of $\mathbb{C}\left[z_{1}, \ldots, z_{r}\right]$ generated by monomials. These ideals are in one-to-one correspondence with $r$-dimensional partitions of $n$. Indeed, given an $r$-dimensional partition $\left(n_{i_{1}, \ldots, i_{r-1}}\right)_{i_{1}, \ldots, i_{r-1} \geq 0}$ of $n$, the ideal of $\mathbb{C}\left[z_{1}, \ldots, z_{r}\right]$ generated by the monomials $z_{1}^{i_{1}} \cdots z_{r-1}^{i_{r-1}} z_{r}^{n_{i_{1}}, \ldots, i_{r-1}}$ has colength- $n$. Conversely, given a colength- $n$ ideal $I$ of $\mathbb{C}\left[z_{1}, \ldots, z_{r}\right]$ generated by monomials, we obtain an $r$-dimensional partition $\left(n_{i_{1}, \ldots, i_{r-1}}\right)_{i_{1}, \ldots, i_{r-1} \geq 0}$ of $n$ by putting

$$
n_{i_{1}, \ldots, i_{r-1}}=\min \left\{i_{r} \mid z_{1}^{i_{1}} \cdots z_{r-1}^{i_{r-1}} z_{r}^{i_{r}} \in I\right\} .
$$

Therefore, by (2.7), we have (see the Proposition 5.1 in [2]):

$$
\chi\left(\operatorname{Hilb}^{n}\left(\mathbb{C}^{r}, O\right)\right)=P_{r}(n) .
$$


When $r=\operatorname{dim}(X)=3$, torus actions on the moduli space $\mathfrak{M}_{n}$ for a toric variety $X$ have been studied in [13]. For $r \geq 3$ and for torus actions on our punctual moduli space $\mathfrak{M}_{n, L, O}^{\mathbb{C}^{r}}$, we choose the line $L$ to be defined by the equations:

$$
z_{1}=\ldots=z_{r-1}=0 .
$$

Then the $\mathbb{C}^{*}$-action (7.4) on $\mathbb{C}^{r}$ induces a $\mathbb{C}^{*}$-action on $\mathfrak{M}_{n, L, O}^{\mathbb{C}^{r}}$. Again, choose the weights $w_{1}, \ldots, w_{r} \in \mathbb{Z}$ in $(7.4)$ properly. Then the $\mathbb{C}^{*}$-fixed points in $\mathfrak{M}_{n, L, O}^{\mathbb{C}^{r}}$ are precisely those corresponding to the ideals $I$ of $\mathbb{C}\left[z_{1}, \ldots, z_{r}\right]$ such that $I$ is generated by monomials, $I \subset\left(z_{1}, \ldots, z_{r-1}\right)$, and

$$
\operatorname{dim}_{\mathbb{C}} \frac{\left(z_{1}, \ldots, z_{r-1}\right)}{I}=n
$$

As in the previous paragraph, we see that these ideals are in one-to-one correspondence with punctual $r$-dimensional partitions of $n$ (note that a linear basis of the ideal $\left(z_{1}, \ldots, z_{r-1}\right)$ consists of all the monomials $z_{1}^{i_{1}} \cdots z_{r-1}^{i_{r-1}} z_{r}^{i_{r}}$ with

$$
\left(i_{1}, \ldots, i_{r-1}\right) \in\left(\mathbb{Z}_{\geq 0}\right)^{r-1}-\{(0, \ldots, 0)\},
$$

and $\left.i_{r} \geq 0\right)$. Therefore, we obtain from (2.7) that

$$
\chi\left(\mathfrak{M}_{n, L, O}^{\mathbb{C}^{r}}\right)=\widetilde{P}_{r}(n) .
$$

Theorem 7.2. Under Assumption 3.1, let $r \geq 2$. Then,

$$
\sum_{n=0}^{+\infty} \chi\left(\mathfrak{M}_{n}\right) q^{n}=\sum_{n=0}^{+\infty} \chi\left(X^{[n]}\right) q^{n} \cdot \chi(S) \cdot\left(\frac{\sum_{n=0}^{+\infty} \widetilde{P}_{r}(n) q^{n}}{\sum_{n=0}^{+\infty} P_{r}(n) q^{n}}\right)^{2-2 g}
$$

Proof. The formula follows from Proposition 6.2, (7.6) and (7.7).

Corollary 7.3. If $\mu: X \rightarrow S$ is an elliptic fibration, then

$$
\sum_{n=0}^{+\infty} \chi\left(\mathfrak{M}_{n}\right) q^{n}=\sum_{n=0}^{+\infty} \chi\left(X^{[n]}\right) q^{n} \cdot \chi(S) .
$$

Conjecture 7.4. Let $r \geq 2$, and let $P_{r}(n)$ and $\widetilde{P}_{r}(n)$ be defined above. Then,

$$
\frac{\sum_{n=0}^{+\infty} \widetilde{P}_{r}(n) q^{n}}{\sum_{n=0}^{+\infty} P_{r}(n) q^{n}}=\frac{1}{(1-q)^{r-2}}
$$


The conjecture is clearly true when $r=2$. The next lemma handles $r=3$.

Lemma 7.5. Conjecture 7.4 holds when $r=3$.

Proof. We shall use notations and results from Section. 11.2 of [1]. Identify our 3-dimensional partitions with the plane partitions there, i.e., our 3 -dimensional partition $\left(n_{i_{1}, i_{2}}\right)_{i_{1}, i_{2} \geq 0}$ is identified with the plane partition whose entry at the lattice point $\left(i_{1}, i_{2}\right), i_{1}, i_{2} \geq 0$ in the plane is equal to $n_{i_{1}, i_{2}}$. Similarly, our punctual 3 -dimensional partitions will correspond to the punctual plane partitions.

Let $\pi_{\ell}\left(n_{1}, \ldots, n_{k} ; q\right)$ be the generating function for plane partitions with at most $\ell$ columns, at most $k$ rows, and with $n_{i}$ being the first entry in the $i$-th row. Then,

$$
\begin{aligned}
& \pi_{\ell+1}\left(n_{1}, \ldots, n_{k} ; q\right) \\
& =q^{n_{1}+\ldots+n_{k}} \sum_{m_{k}=0}^{n_{k}} \sum_{m_{k-1}=m_{k}}^{n_{k-1}} \ldots \sum_{m_{1}=m_{2}}^{n_{1}} \pi_{\ell}\left(m_{1}, \ldots, m_{k} ; q\right) .
\end{aligned}
$$

by the formula (11.2.1) in [1]. Let $S_{k, \ell}(m, n)$ (respectively, $\left.\widetilde{S}_{k, \ell}(m, n)\right)$ denote the set of plane partitions (respectively, punctual plane partitions) of $m$ with at most $\ell$ columns, at most $k$ rows, and with each entry $\leq n$. Let $p_{k, \ell}(m, n)=$ $\left|S_{k, \ell}(m, n)\right|$ and $\tilde{p}_{k, \ell}(m, n)=\left|\widetilde{S}_{k, \ell}(m, n)\right|$. Define two generating functions:

$$
\begin{aligned}
& \pi_{k, \ell}(n ; q)=\sum_{m=0}^{+\infty} p_{k, \ell}(m, n) q^{m} \\
& \tilde{\pi}_{k, \ell}(n ; q)=\sum_{m=0}^{+\infty} \tilde{p}_{k, \ell}(m, n) q^{m} .
\end{aligned}
$$

So $\pi_{+\infty,+\infty}(+\infty ; q)=\sum_{m=0}^{+\infty} P_{3}(m) q^{m}$ and $\tilde{\pi}_{+\infty,+\infty}(+\infty ; q)=\sum_{m=0}^{+\infty} \widetilde{P}_{3}(m) q^{m}$.

Define

$$
(q)_{i}=(1-q)\left(1-q^{2}\right) \cdots\left(1-q^{i}\right)
$$

for positive integers $i$. By the Theorem 11.2 in [1],

$$
\pi_{k, \ell}(n ; q)=\frac{(q)_{1}(q)_{2} \cdots(q)_{k-1}}{(q)_{\ell}(q)_{\ell+1} \cdots(q)_{\ell+k-1}} \cdot \frac{(q)_{n+\ell}(q)_{n+\ell+1} \cdots(q)_{n+\ell+k-1}}{(q)_{n}(q)_{n+1} \cdots(q)_{n+k-1}} .
$$


Let $\tilde{\lambda} \in \widetilde{S}_{k, \ell}(m, n)$. By placing $n$ at the origin of the plane, we obtain $\lambda \in S_{k, \ell}(m+n, n)$. Conversely, if $\lambda \in S_{k, \ell}(m+n, n)$ and if the part of $\lambda$ at the origin is $n$, then by deleting the part at the origin, we obtain $\tilde{\lambda} \in \widetilde{S}_{k, \ell}(m, n)$. Hence

$$
\tilde{\pi}_{k, \ell}(n ; q)=q^{-n} \sum_{m_{k} \leq \ldots \leq m_{2} \leq n} \pi_{\ell}\left(n, m_{2}, \ldots, m_{k} ; q\right) .
$$

Setting $n_{1}=\ldots=n_{k}=n$ in $(7.10)$, we conclude that

$$
\begin{aligned}
\pi_{\ell+1}(\underbrace{n, \ldots, n}_{k \text { copies }} ; q) & =q^{k n} \sum_{m_{k} \leq \ldots \leq m_{2} \leq m_{1} \leq n} \pi_{\ell}\left(m_{1}, \ldots, m_{k} ; q\right) \\
& =q^{k n} \sum_{m_{1}=0}^{n} \sum_{m_{k} \leq \ldots \leq m_{2} \leq m_{1}} \pi_{\ell}\left(m_{1}, m_{2}, \ldots, m_{k} ; q\right) .
\end{aligned}
$$

Combining this with formula (7.13), we see that

$$
\pi_{\ell+1}(\underbrace{n, \ldots, n}_{k \text { copies }} ; q)=q^{k n} \sum_{m_{1}=0}^{n} q^{m_{1}} \cdot \tilde{\pi}_{k, \ell}\left(m_{1} ; q\right) .
$$

On the other hand, by the formula (11.2.8) in [1],

$$
\pi_{\ell+1}(\underbrace{n, \ldots, n}_{k \text { copies }} ; q)=q^{k n} \cdot \pi_{k, \ell}(n ; q) .
$$

Therefore, we see immediately from (7.14) that

$$
\pi_{k, \ell}(n ; q)=\sum_{m_{1}=0}^{n} q^{m_{1}} \cdot \tilde{\pi}_{k, \ell}\left(m_{1} ; q\right) .
$$

Thus, $\pi_{k, \ell}(n ; q)-\pi_{k, \ell}(n-1 ; q)=q^{n} \cdot \tilde{\pi}_{k, \ell}(n ; q)$, i.e.,

$$
\tilde{\pi}_{k, \ell}(n ; q)=q^{-n}\left[\pi_{k, \ell}(n ; q)-\pi_{k, \ell}(n-1 ; q)\right] .
$$

To take the limits $k, \ell, n \rightarrow+\infty$, we assume $|q|<1$ in the rest of the proof. By (7.15), (7.12) and the definition of $(q)_{i}$ from $(7.11)$, we have

$$
\begin{aligned}
& \tilde{\pi}_{k, \ell}(n ; q) \\
= & \pi_{k, \ell}(n-1 ; q) \cdot \frac{1}{q^{n}}\left\{\frac{\left(1-q^{n+\ell}\right)\left(1-q^{n+\ell+1}\right) \cdots\left(1-q^{n+\ell+k-1}\right)}{\left(1-q^{n}\right)\left(1-q^{n+1}\right) \cdots\left(1-q^{n+k-1}\right)}-1\right\} \\
= & \pi_{k, \ell}(n-1 ; q) \cdot \frac{1+q+\cdots+q^{k-1}+O\left(q^{n}\right)+O\left(q^{\ell}\right)}{\left(1-q^{n}\right)\left(1-q^{n+1}\right) \cdots\left(1-q^{n+k-1}\right)} .
\end{aligned}
$$


Now taking the limit $\ell, n \rightarrow+\infty$, we conclude that

$$
\tilde{\pi}_{k,+\infty}(+\infty ; q)=\pi_{k,+\infty}(+\infty ; q) \cdot\left(1+q+\cdots+q^{k-1}\right) .
$$

Finally, letting $k \rightarrow+\infty$, we immediately obtain

$$
\tilde{\pi}_{+\infty,+\infty}(+\infty ; q)=\pi_{+\infty,+\infty}(+\infty ; q) \cdot \frac{1}{1-q},
$$

i.e., $\sum_{m=0}^{+\infty} \widetilde{P}_{3}(m) q^{m}=\sum_{m=0}^{+\infty} P_{3}(m) q^{m} \cdot \frac{1}{1-q}$. This proves $(7.9)$ for $r=3$.

Corollary 7.6. Under Assumption 3.1 , let $2 \leq r \leq 3$. Then,

$$
\sum_{n=0}^{+\infty} \chi\left(\mathfrak{M}_{n}\right) q^{n}=\sum_{n=0}^{+\infty} \chi\left(X^{[n]}\right) q^{n} \cdot \chi(S) \cdot \frac{1}{(1-q)^{(r-2) \cdot(2-2 g)}}
$$

Proof. Follows immediately from Theorem 7.2 and Lemma 7.5.

\section{Acknowledgments.}

The authors are grateful to Professors Robert Friedman, Sheldon Katz and Jun Li for valuable helps and stimulating discussions. The first author thanks the Mathematics Department at the University of Missouri for the hospitality and the support during his visit in January of 2005. Thanks also go to the referee for suggesting improvements.

\section{References.}

[1] G. Andrews, The Theory of Partitions, Encyclopedia of Mathematics and its Applications (1976).

[2] J. Cheah, On the cohomology of Hilbert schemes of points, J. Alg. Geom. 5 (1996), 479-511.

[3] V. I. Danilov, A. G. Khovanskii, Newton polyhedra and an algorithm for computing Hodge-Deligne numbers, Math. USSR Izv. 29 (1987), 279-298.

[4] P. Deligne, Théorie de Hodge III, I.H.E.S. Publ. Math. 44 (1974), 5-77. 
[5] S. K. Donaldson, R. P. Thomas, Gauge theory in higher dimensions, In: The geometric universe (Oxford, 1996), 31-47. Oxford University. Press, Oxford, 1998.

[6] W. Fulton, Introduction to toric varieties, Annals of Mathematics Studies 131, Princeton University Press, Princeton, 1993.

[7] L. Göttsche, The Betti numbers of the Hilbert scheme of points on a smooth projective surface, Math. Ann. 286 (1990), 193-207.

[8] L. Göttsche, Hilbert schemes of zero-dimensional subschemes of smooth varieties, Lecture Notes in Mathematics 1572, Springer-Verlag, Berlin, 1994.

[9] A. Grothendieck, Techniques de construction et théorèmes d'existence en géométrie algébrique IV, Sem. Bourbaki 221, 13. 1960-1961.

[10] D. Huybrechts, M. Lehn, The geometry of moduli spaces of sheaves, Aspects of Mathematics, E31. Friedr. Vieweg \& Sohn, Braunschweig, 1997.

[11] S. Katz, Gromov-Witten, Gopakumar-Vafa, and Donaldson-Thomas invariants of Calabi-Yau threefolds, Preprint.

[12] H. B. Lawson, Jr., S.S.T. Yau, Holomorphic symmetries, Ann. Sci. Ecole Norm. Sup., 4e série, t. 20 (1987), 557-577.

[13] D. Maulik, N. Nekrasov, A. Okounkov, R. Pandharipande, GromovWitten theory and Donaldson-Thomas theory, Preprint.

[14] D. Maulik, N. Nekrasov, A. Okounkov, R. Pandharipande, GromovWitten theory and Donaldson-Thomas theory, II, Preprint.

[15] D. Maulik, R. Pandharipande, Foundation of Donaldson-Thomas theory, In preparation.

[16] J.S. Milne, Étale cohomology. Princeton Math. Series 33. Princeton: Princeton University Press 1980.

[17] A. Okounkov, R. Pandharipande, Quantum cohomology of the Hilbert schemes of points in the plane, math.AG/0411210.

[18] R. P. Thomas, A holomorphic Casson invariant for Calabi-Yau 3-folds, and bundles on K3 fibrations, J. Differential Geom. 54 (2000), 367-438. 